provides concrete evidence that fatigue factors are predictor to near misses and not in the case of accidents. The introduction of post-prevention control however proven to be effective with workers' fatigue and emotional exhaustion instead of working condition of this companies. This study contributes to aircraft ground handling safety by investigating the workers' fatigue factors, thus helping to improve the company and individual performance as well as reduce the risks related to fatigue.

\section{C.006 KEY FACTORS FOR SUCCESSFUL IMPLEMENTATION, MAINTENANCE, AND SUSTAINABILITY OF WORK SAFETY INTERVENTIONS}

${ }^{1}$ Neil Kirby*, ${ }^{1}$ Julia Harries, ${ }^{2}$ Jerry Ford. ${ }^{1}$ The University of Adelaide, Adelaide, Australia; ${ }^{2}$ Flinders University, Bedford Park, Australia

10.1136/injuryprev-2021-safety.201

Background An investigation of Disability Support Workers' (DSWs) psychosocial work safety showed poorer health and safety outcomes than norm groups. Numerous safety interventions derived from a work safety climate measure and stakeholder feedback were identified, with seven translated into practice and evaluated. At evaluation, roll-out varied from organisation-wide implementation to implementation that was restricted to limited work-sites. Successful implementation of interventions was facilitated by wide safety benefit consensus, strong overt management commitment, and use of change champions. Findings supported the importance of factors identified in research for successful change implementation, but less research had been reported about ensuring longer-term intervention maintenance and/or sustainability. Thus, a followup study used and evaluated a model to understand the key factors required for the successful implementation, maintenance and sustainability of work safety interventions.

Method Semi-structured interviews were used to follow the seven implemented work safety interventions to identify factors facilitating or hindering their implementation, maintenance, and sustainability.

Results Numerous influential intervention and organisational factors were identified. These were conceptualised in the model as: 1) intervention attributes; 2) implementation process characteristics; 3) leadership behaviours and characteristics; and 4) the internal and external organisational context.

Conclusions Findings suggested no 'one size fits all' approach to factors critical for implementing, maintaining and/or sustaining these successful interventions. Rather, relevant factors and combinations of factors varied in importance across interventions and over the course of the intervention implementation.

Learning Outcomes Findings support the need to identify and address factors facilitating and/or hindering the longer-term sustainability of implemented safety recommendations.

\section{C.007 IMPROVING THE SAFETY OF VULNERABLE GARMENT SECTOR WORKERS COMMUTING TO WORK}

${ }^{1}$ Pagna Kim*, ${ }^{1}$ Linh PHAM, ${ }^{1}$ Damon Rusdon, ${ }^{1}$ Piseth IM, ${ }^{2}$ Louise Goldman. ${ }^{1}$ AIP Foundation, Tuol Svay Prey I Commune, Boeung Keng Kang District, Cambodia; ${ }^{2}$ Solidarity Center, Boeung Kak III Commune, Tuol Kork District, Cambodia

10.1136/injuryprev-2021-safety.202
Context In Cambodia, more than 700,000 factory workers face unacceptable and preventable risks daily commuting to \& from work. Irrespective of a number of interventions implemented to mitigate these issues, daily commuting safety risks for factory workers remain high.

Process Our recently completed pilot program focused on four key areas:

1. Road Safety Management

2. Vehicles \& Modes of Transport

3. Safer Infrastructure

4. Safer Road Users

Our activities compliments Universal Road Safety Goals and aligns with the following UN SDGs:

- Good Health

- Sustainable Cities and Communities

Our Program impacted 26,000 workers and drivers and engaged over 100,000 people through online campaigns.

Analysis Gathering data by various methods including worker and driver baseline and end-line KAP surveys, helmet observations and crash reporting facilitated evidence-based decision making and the evaluation of the effectiveness of the program.

Outcomes The Program has made tangible changes to the attitudes and behavior of workers and drivers. $85 \%$ of workers and $28 \%$ of drivers at targeted factories reported safer driving conditions. Reports indicate drink-driving among transport providers reduced by $22 \%$ and speeding reduced by $18 \%$. Helmetwearing at five factories increased by $24 \%$. Passenger vehicle changes at 3 factories total 15 new buses and 30 new vans. Factories report a $77 \%$ decline in the number of crashes.

Learning Outcomes The success of the program confirms the effectiveness of our program model in improving commuting safety among workers in low and middle-income countries. Moreover, lessons learned provide opportunities for improvement and effective adaption to different contexts.

\section{C.008 ACTIVELY ENGAGING ACADEMICS THROUGH AN OHS HAZARD PROFILING EXERCISE: LESSONS LEARNED}

Brendan Henderson*, Ryan Pane. Deakin University, Burwood, Australia

\subsection{6/injuryprev-2021-safety.203}

Context In 2019, Occupational Health and Safety (OHS) staff within the Faculty of Health performed a hazard mapping exercise as a means to greater understand the current OHS risk profile of our teaching operations.

Process Fifteen hazard profiles were performed across five schools within the Faculty of Health. The use of a specifically designed OHS hazard mapping toolkit provided a consultative framework to openly discuss with staff current practices and concerns relative to OHS and teaching program quality.

Analysis A qualitative analysis of the hazards and considerations required to manage student hazards and risks aligned to teaching and research activities was performed though discussion held. Action plans from the common themes identified were implemented with shared responsibility between professional OHS and teaching program staff.

Outcomes The hazard mapping toolkit enabled a consultative and stepped approach to encourage open discussion, and aid greater understanding of the OHS responsibilities relative to a teaching program domain. 
Learning Outcomes Undertaking an OHS risk management approach with academic staff was a positively received experience that provided an opportunity to greater understand teaching program hazards. A consultative approach aided the education of teaching staff around their OHS roles and responsibilities, inclusive of applying a reasonably practicable approach to risk mitigation from identified hazards. Finally, the toolkit saw transferability so that a quality teaching program lens was able to be applied throughout discussions and action plan implementation.

\section{E-Posters P4 - Child, Community, Sport, Falls, Strategy, Systems \& WHS, March 25, 2021}

\section{P4.001 EFFECTIVENESS OF THE CHILD BURN INJURY PREVENTION CAMPAIGN, IN MONGOLIA}

'Gerelmaa Gunsmaa*, 'Ichikawa Masao, ${ }^{2}$ Haruhiko Inada, ${ }^{3}$ Badarch Tumen Ulzi. ${ }^{1}$ Department of Global Public Health, Faculty of Medicine, University of Tsukuba, Tsukub, Japan; ${ }^{2}$ Department of International Health, Jonhs Hopkins Baloomberg School of Public Health, Baltimore, USA; ${ }^{3}$ Department of Statistics and Surveylliance, National Trauma and Ortopedic Research Center, Ullaanbaatar, Mongolia

\subsection{6/injuryprev-2021-safety.204}

Introduction Global Burden of Disease Study 2013 demonstrated that in Mongolia, child burn mortality is remarkably high, which is 8.1 per 100,000 children aged $<15$ years as compared with global rate 2.5. To tackle this problem, nationwide burn prevention campaigns were implemented from August 2014 to May 2015 and from January 2017 to February 2017 in Mongolia.

Methods To evaluate the campaign effectiveness, we conducted an interrupted time-series analysis, using the monthly rate from 2009 to 2018 of child burn injuries per 10,000 by sex, age group, mode and severity of burn injury. The burn injury data were derived from the National Trauma and Orthopedic Research Centre.

Results During the study period, there were 27800 medically attended child burn injuries, 34\% of them were major burns. After the first campaign, the rate of scalds shortly decreased compared with that of other types of burns. The decreasing trend was significant among children aged $<4$ compared with children aged from 5 to 14 , and the relative change of the scald rate was $-29 \%$ in one-year post-intervention. However, the rate of major burns continued to increase irrespective of the types of burns. After the second campaign started, none of the rates declined.

Conclusions Though the first campaign was effective in reducing minor and moderate scalds among young children, major burns did not decrease. The importance of understanding the success and failure of the prevention campaign is to help to integrate burn prevention.

\section{P4.002 COMMUNITY ENGAGEMENT IN WEB-DESIGN TO PROMOTE SAFE RECREATION FOR CHILDREN WITH AUTISM}

${ }^{1}$ Crystal A Shannon*, ${ }^{1}$ Lise L Olsen, ${ }^{1}$ Rachelle Hole, ${ }^{2}$ Grace larocci, ${ }^{3}$ Mariana Brussoni, ${ }^{4}$ Stephanie Jull. 'University of British Columbia, Okanagan, Kelowna, Canada; ${ }^{2}$ Simon Fraser University, Burnaby, Canada; ${ }^{3}$ University of British Columbia, Vancouver, Canada; ${ }^{4}$ Canucks Autism Network, Vancouver, Canada

10.1136/injuryprev-2021-safety.205
Background Participation in recreational activity by children with autism is important for health and social inclusion. However, these children and their families experience many barriers to recreation participation including parental concerns about safety and injury risks and a lack of educational resources to address children's recreational safety needs. In this study, we are aiming to design a web-based parent resource to increase safety awareness and promote appropriate strategies for safe recreation.

Methods Using a community-based research approach, this study will engage parents and stakeholders in urban and rural settings to design a website to meet the needs of families of children with autism. Focus groups, interviews and survey methods will be used to evaluate the development, feasibility and effectiveness of the website resource.

Results Findings from parent focus groups conducted in urban and rural communities in British Columbia, Canada will be presented. These results will be used to identify priority website components to inform the blueprint for the website structure, content and design. The website design process and testing procedures will be highlighted.

Conclusion Community engagement is needed to identify unique safety needs and strategies to foster safe active recreation for children with autism. This research supports SDG \#10.2 to reduce inequalities through better understanding to promote recreational inclusion for families and children living with autism.

Learning Outcomes Participants will gain: 1) awareness of family-centred priority safety issues and strategies and 2) appreciation of how community engagement approaches can inform website design to promote safe recreation for children with autism.

\section{P4.003 THE COMPLEXITY OF SUPERVISION: IMPACT OF PARENTAL SUPERVISION STYLE ON CHILDREN'S RISK-TAKING}

${ }^{1}$ Casie Morgan*, ${ }^{1}$ Claire Estep, ${ }^{2}$ Caitlin Bullard, ${ }^{3}$ Brittany Cangialosi, ${ }^{1}$ David Schwebel. ${ }^{1}$ University of Alabama At Birmingham, Birmingham, USA; ${ }^{2}$ Baylor University, Waco, USA; ${ }^{3}$ Jacksonville State University, Jacksonville, USA

\subsection{6/injuryprev-2021-safety.206}

Background Active supervision to prevent child injury encompasses close proximity, full attention, and continuous monitoring (Saluja et al, 2004). Absent components may increase injury risk. We examined the impact of supervision and supervision styles on children's risk-taking.

Methods 59 children aged 4-6 years (Mage $=5.47, \mathrm{SD}=.47$ ) engaged in an activity room with hazards (e.g., ball pit) for ten minutes (supervised, then unsupervised). Parents completed questionnaires while 'supervising,' creating poor attention/noncontinuous monitoring. Children also responded to 12 illustrated risk-taking activities (6 pairs, split between unsupervised/actively supervised), indicating preferred engagement in risks.

To assess supervision style, parents completed the PSAPQ (Morrongiello \& House, 2004), which includes four scales: supervision (keep close watch), protectiveness (strong sense of responsibility), fate (injuries are bad luck; excluded from analyses given focus), and risk-tolerance (encourage independence). 